\section{PSICOLOGIA IBEROAMERICANA}

Psicología Iberoamericana

ISSN: 1405-0943

psicología.iberoamericana@uia.mx

Universidad Iberoamericana, Ciudad de

México

México

\title{
Björne, Magnus
}

Una Perspectiva Psicoecducativa del Autismo a lo Largo de la Vida

Psicología lberoamericana, vol. 14, núm. 1, 2006, pp. 21-26

Universidad Iberoamericana, Ciudad de México

Distrito Federal, México

Disponible en: http://www.redalyc.org/articulo.oa?id=133926960004

- Cómo citar el artículo

- Número completo

- Más información del artículo

- Página de la revista en redalyc.org

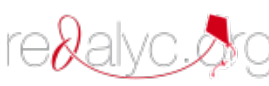

Sistema de Información Científica

Red de Revistas Científicas de América Latina, el Caribe, España y Portugal

Proyecto académico sin fines de lucro, desarrollado bajo la iniciativa de acceso abierto 


\title{
Una Perspectiva Psicoeducativa del Autismo a lo Largo de la Vida A Lifelong Psychoeducational Perspective on Autism*
}

\author{
Magnus Björne*** \\ Centro Nimbosgarden de Autismo, Suecia
}

\section{Resumen}

En el tratamiento del niño con autismo resulta esencial tener una perspectiva de vida, si es que se pretende proporcionar a este niño los medios necesarios para convertirse en un adulto que viva con la mayor calidad de vida posible. Una perspectiva de toda la vida implica que desde la infancia se intente formar una imagen del adulto. Es un arduo trabajo, pero no es una misión imposible. Aún más, no sólo resulta alentador para el niño, es estimulante también para los padres y los profesionales involucrados. Se revisan los conceptos de normalidad, calidad de vida, así como el derecho del niño a un programa educativo individual.

Deseriptores: autismo, tratamiento psicoeducativo, calidad de vida, normalidad, derechos del niño

\begin{abstract}
In the treatment of a child with autism it is essential to have a life-long perspective if we want to provide this child with the necessary means to become an adult with the best possible quality of life. To ensure that a child with autism can achieve their maximum level of quality of life, we have to look to the future and take a very long-term view. A life-long perspective implies that right from infancy we try to shape an image of the adult. It is a hard job but not impossible. Moreover it is not only encouraging for the child, but is also stimulating for the parents and professionals involved. The concepts of normality and quality of life are reviewed, as well as the right of the child to an individual education program.

Key words: Autism, psycho-educational treatment, quality of life, normality, children's rights
\end{abstract}

\section{Introducción}

Para que el tratamiento de personas con cuadro autista sea exitoso debe tomarse en consideración el concepto calidad de vida. Es un concepto complejo y se desviaria el propósito de este trabajo si se intentara dar una definición de su significado para una persona con autismo. Debido a que el concepto es definido subjetivamente, probablemente lo más esencial seria responder a la pregunta: ¿qué es lo que este niño -o este adulto- necesita saber o aprender para mejorar su calidad de vida? La pregunta parece sencilla; sin embargo su respuesta requiere una larga discusión. Si no existe un entendimiento profundo sobre el autismo y sobre cómo esta discapacidad repercute en la personalidad, resulta imposible responder a esta in- terrogante y proporcionar el apoyo necesario para que la persona con autismo alcance el grado más alto de calidad de vida que pueda obtener. Es decir, un tratamiento exitoso y efectivo necesariamente debe estar fundamentado en el conocimiento y entendimiento del autismo. Más adelante enumeraré algunas de las habilidades que deberian desarrollarse en las personas con este trastorno, pero primero es necesario discutir sobre el autismo en general, así como acerca de algunos conceptos clave cuando se habla de calidad de vida.

\section{El aprendizaje en el autismo}

Hace 15 años la discusión sobre el aprendizaje en el autismo en Suecia se basó en un libro titulado La pe-

\footnotetext{
*Agradecemos la colaboración de Karina Lozano Aguirre en la traducción de este artículo.

*Contacto:magnus@nimpius.se
} 
dagogia de las diez mil veces. Los autores plantearon que los niños con autismo aprenden muy despacio y que probablemente requieren repetir una tarea diez mil veces antes de poder asimilarla realmente. Considero que ésta es la manera incorrecta de afrontar la situación. Primero que nada, los niños con autismo no necesariamente aprenden lentamente; aprenden de manera distinta; por lo que tenemos que adaptar las estrategias de aprendizaje a los niños y no intentar que éstos embonen en el molde de aprendizaje normal. Esta conclusión también aplica para los adultos con autismo.

Existe una conexión estrecha entre aprendizaje y cognición. Yo afirmo que las personas con autismo aprenden de una manera diferente debido a que poseen una cognición cualitativamente distinta. Cualitativamente diferente no necesariamente significa que las personas con autismo desarrollan sus habilidades cognitivas más lento que otras personas o que carecen de ciertas habilidades o caracteristicas. Cualitativamente diferente significa principalmente que las personas con autismo poseen ciertas características que otros individuos no poseen; esto significa que existen ciertas conductas exclusivas del autismo. De aquí la importancia de entender cómo la cognición en el autismo difiere de la cognición típica antes de empezar a pensar en el tratamiento y en qué habilidades requieren aprender las personas con este trastorno.

$\mathrm{Ni}$ el criterio diagnóstico ni otras conductas especificas del autismo dan una respuesta a la pregunta de cómo se estructura la cognición en el autismo. No basta con enfocarse sólo en las conductas especificas, es necesario ahondar más allá de la superficie de estas conductas para ser capaces de explicar por qué aparecen y poder formarnos una imagen de cómo las habilidades cognitivas en el autismo difieren de las tipicas. Una forma de lograrlo puede ser escuchando a las personas con autismo que son capaces de explicar su manera de pensar.

Muchos "reportes desde el interior del autismo" hacen énfasis en la percepción diferente, en cómo esta hipo o hipersensibilidad frente a los estimulos acarrea grandes dificultades en la vida cotidiana y en la interacción social. Actualmente, las experiencias sensoriales diferentes son vistas como un importante indicio temprano de autismo. Estudios con infantes han subrayado no solamente la hipo e hipersensibilidad hacia los estimulos de los niños que posteriormente han sido diagnosticados con autismo, sino también las dificultades que éstos presentan en orientar su atención a estimulos sociales y no sociales, asi como im- pedimentos motrices. Se ha visto que estas dificultades pueden presentarse a una edad de seis meses $\mathrm{y}$ al parecer aun a más temprana edad.

Las experiencias sensoriales, la atención y las destrezas motrices, son centrales en todo aprendizaje e influyen en la experimentación del mundo. Si el niño presenta dificultades en estas áreas, esto afectará todo su desarrollo. Con diferentes experiencias sensoriales, una manera distinta de atender y una forma disimil de moverse y utilizar el cuerpo, las habilidades cognitivas se desarrollarán de una manera cualitativamente diferente. Jim Sinclair, quien tiene autismo, describe esta situación de una forma muy ilustrativa:

[el autismo] es penetrante; da color a cada experiencia, a cada sensación, percepción, pensamiento, emoción, encuentro y a cada aspecto de la existencia. No es posible retirar el autismo de la persona, y si fuera posible, la persona que resultara no seria la misma persona con la que se inició (Sinclair, 1993).

Su conclusión es que el autismo es una forma de ser, no algo que una persona padece.

Un tratamiento efectivo debe estar basado en este conocimiento. Las estrategias para el aprendizaje tienen que construirse sobre las fortalezas y con el propósito de compensar las debilidades. Generalmente las personas con autismo tienen relativas fortalezas, como por ejemplo habilidades visuales, habilidades para reconocer detalles y de memorización, las cuales se pueden convertir en las bases de un funcionamiento adulto exitoso. Además, las estrategias para el aprendizaje tienen que compensar las debilidades, tales como las dificultades para la organización, la secuenciación y la generalización en la ejecución de tareas dentro de un contexto; así como problemas con la concentración, la atención y la búsqueda de significado.

Esto nos hace reflexionar: si las personas con autismo tienen una cognición completamente distinta, se tienen que adaptar a mucho más que sólo al proceso enseñanza-aprendizaje. Probablemente la normalidad es otra si uno tiene autismo. Jim Sinclair opina que: "Lo que es normal para otra persona para mi no lo es, y los que es normal para mi no lo es para los otros" (Sinclair, 1992). Si esta es una suposición correcta tendremos que contestar a la interrogante: si se toma en cuenta la normalidad, ¿quién está dentro de la normalidad? o ¿los que no tenemos autismo, tenemos el derecho de imponer nuestra normalidad sobre las personas con autismo? 


\section{La normalidad como meta}

Estas preguntas son rara vez formuladas en el campo del autismo. Existen muchos y diversos tratamientos que dan por sentado la normalidad. Es decir, estos tratamientos, si bien son bastante heterogéneos, tienen en común que suponen que las personas con autismo tienen una cognición normal. Algunos de estos tratamientos, que incluyen por ejemplo Terapia de Contención (Holding Therapy) y de Facilitación de la comunicación, se construyen a partir de la suposición de que el autismo es un caparazón que se sitúa entre el saludable Yo interno de la persona y el mundo externo. La meta de estos tratamientos es liberar a la persona atrapada en esa concha, una persona normal con habilidades cognitivas normales. Desde su punto de vista, sólo es cuestión de romper la dura concha.

Ivar Lovaas y defensores de ABA (Applied Behavior Analysis o Análisis de la Conducta Aplicado) son ejemplos de otro enfoque de la normalización. Ellos no asumen que las personas con autismo tienen una cognición normal, sino que buscan enseñar a los niños con autismo a actuar normalmente. Desde su perspectiva, la mejor evidencia de un buen tratamiento es una conducta normal. En los años sesenta, Lovaas utilizó estímulos aversivos, aun incluyendo los electroshocks para suprimir conductas inaceptables y para facilitar el aprendizaje de las conductas aceptables, es decir, normales (Simmons III \& Lovaas, 1969). Desde entonces sus métodos han cambiado, pero aún ABA busca la misma meta: enseñar a los niños con autismo a actuar normalmente.

\section{Un enfoque diferente de normalidad}

El programa TEACCH (Tratamiento y Educación de Niños con Autismo y otros Trastornos de la Comunicación), defiende un tercer y casi opuesto abordaje del concepto de normalización. Se desarrolló en la Universidad de Carolina del Norte, Estados Unidos, alrededor de los años setenta y tal vez es famoso por su pedagogía y la estructura de enseñanza; pero el programa TEACCH es más que un método o una técnica: es una filosofia, una forma de mirar al autismo, $y$ por supuesto, una forma de encontrarse con los individuos con autismo y sus familias.
El programa TEACCH defiende la postura de que las personas con autismo tienen su propia cultura, y que tienen el derecho de tenerla. El punto es que si aceptamos que el autismo tiene una causa biológica, una causa que lleva a la persona a tener una forma distinta de percibir al mundo, tenemos que tomar este argumento seriamente y llegar a conclusiones correctas: una percepción diferente lleva a una cognición distinta, y esto nos da como resultado valores disimiles. Si aceptamos esta conclusión, tenemos que acercarnos a las personas con autismo como nos acercamos a personas de diferentes culturas: respetando sus valores, su forma de pensar y su manera de vivir.

Considero que la División TEACCH tiene un fuerte argumento a este aspecto. Desde mi punto de vista actuar normalmente no puede ser una meta en sí misma. Estoy convencido de que nosotros, que no tenemos autismo, no tenemos ningún derecho a forzar a las personas con autismo a nuestra normalidad. Además, no existen argumentos sólidos para la normalización. La normalización podría llegar a ser una meta siempre y cuando respetemos la disimilitud cognitiva del autismo. En ciertas situaciones una conducta normal puede traer consecuencias positivas (por ejemplo, una actuación normal puede facilitar el paso entre la gente en un centro comercial), y algunas personas con un autismo altamente funcional pueden desear ser normales. Para ellos, actuar normalmente puede ser una forma de ser aceptado como miembro de la comunidad. En años recientes, el movimiento del Empoderamiento ha optado por otra perspectiva: Yo tengo autismo, yo soy diferente, y exijo ser aceptado como soy.

Esta perspectiva nos lleva a un concepto clave en el programa TEACCH: compromiso. Tratar a alguien con respeto no quiere decir que una de las dos partes tenga el privilegio de imponer las reglas. Respetar implica construir un puente entre dos culturas. Nosotros, que pertenecemos a la cultura no autista, tenemos la responsabilidad de facilitar que las personas con autismo puedan vivir y formar parte de la comunidad, de hacer que la comunidad sea accesible para ellos. Por supuesto, con el máximo respeto hacia su forma de vivir.

\section{Implicaciones pedagógicas}

Habiendo convenido que las personas con autismo tienen una cognición distinta y que tienen su propia cul- 
tura, es tiempo de regresar a la interrogante: ¿qué es lo que las personas con autismo necesitan saber o aprender para lograr una mejor calidad de vida? Aquí me concentraré en la transición a la adultez del grupo de niños con autismo y retraso mental. Es importante enfatizar que aun los individuos con retraso mental severo pueden alcanzar un grado relativamente alto de calidad de vida.

Voy a utilizar el Centro de Autismo Nimbüsgarden en Suecia como un ejemplo. Nimbüsgarden es una corporación privada que ofrece cuatro tipos de servicio: una casa-hogar para un grupo de adultos, un centro de actividades diarias, una estancia temporal para niños y adolescentes, y una unidad de educación y consulta. Nuestra base pedagógica se define mediante dos condiciones diferentes.

La primera condición es la legislación. En Suecia el Acta referente al apoyo y servicio para personas con cierto grado de deterioro funcional (Swedish Code of Statutes SFS, 1993:387) brinda a las personas con discapacidades ciertos derechos. El acta se obliga a "...promover equidad en las condiciones de vida y la completa participación en la vida de la comunidad". Los servicios "...se obligan a adaptarse a las necesidades del individuo receptor y a establecerse de tal forma que sean accesibles para aquellos que las necesitan, asi como a fomentar la habilidad... de vivir una vida independiente".

La segunda condición es el programa TEACCH. Nimbüsgarden es uno de los numerosos servicios en Suecia que están afiliados a la División TEACCH, en Carolina del Norte. Esto quiere decir que existe un supervisor que nos visita por lo menos una vez al año $y$ que nuestro trabajo tiene que seguir ciertas recomendaciones. No es suficiente usar la enseñanza estructurada en el trabajo diario. Nuestro trabajo tiene que estar basado en toda la filosofia TEACCH, incluyendo la misión TEACCH: "Capacitar individuos con autismo para funcionar tan significativa e independientemente como sea posible dentro de la comunidad" (www.teacch.com).

\section{Conceptos clave de calidad de vida}

Como se puede observar, existen muchas similitudes entre la misión de TEACCH y la legislación sueca. Las dos enfatizan en el individuo, en la posibilidad de participar en la comunidad y en el derecho a una vida independiente. Además, la misión TEACCH enfatiza el derecho a llevar una vida significativa.
Yo creo que significativo e independiente son los dos conceptos más interesantes cuando se habla de calidad de vida para las personas con autismo. Debido a que TEACCH defiende el derecho de las personas con autismo a tener su propia cultura, la misión TEACCH, y especialmente estos dos conceptos, dan una dirección firme cuando estamos a punto de contestar la pregunta acerca de lo que un niño con autismo requiere saber para mejorar su calidad de vida. La respuesta tiene que emanar del individuo y sus propios intereses, fortalezas y necesidades. Lo que resulta significativo para una persona no necesariamente lo es para otra, y el más alto grado de independencia no puede ser el mismo entre el grupo de personas con autismo. Esto es, la calidad de vida tiene que ser definida individualmente y por tal motivo el programa educacional individual diferirá de una persona a otra.

\section{Una perspectiva de toda la vida}

El concepto de calidad de vida requiere una perspectiva de toda la vida. La importancia de tomar una perspectiva de este tipo cuando se trabaja con personas con autismo no debe de ser sobreestimada. La perspectiva de toda la vida es necesaria -pero no es suficientecuando se aspira a proveer a un niño con autismo de los medios necesarios para convertirse en un adulto que viva con la mayor calidad de vida posible.

Para tener éxito en este trabajo debe de existir una estrecha colaboración entre todos los involucrados con el niño y su desarrollo. El programa TEACCH subraya la importancia de involucrar a las familias en el tratamiento. Los padres son tomados como coterapeutas y los mejores defensores del niño (Schopler, Mesibov, Shigley \& Bashford, 1984). Como coterapeutas, los padres están profundamente involucrados tanto en la planeación como en el entrenamiento diario. Pero la cooperación entre los profesionales también es fundamental. El profesional debe de tener una visión holística y una perspectiva de toda la vida del niño. Existen muchos niños con autismo que no han alcanzado el mayor grado de calidad de vida posible sólo porque los profesionales, quienes supuestamente deben apoyarlos, no lo han sabido hacer.

Cada niño tiene derecho a un programa individual de educación. Las prioridades van a cambiar a lo largo de la vida pero el niño se beneficiará mucho si las metas principales permanecen constantes. Es 
decir, si se empieza a planear una vida adulta independiente desde los años preescolares y se trata de mantener ese plan (si continúa siendo apropiado) el niño tiene mejores posibilidades como adulto. Una perspectiva de toda la vida nos ayuda a apegarnos a los objetivos principales y a enseñarle al niño habilidades funcionales, es decir, habilidades que son tan necesarias y útiles en la vida diaria del niño como del adulto. Sin una perspectiva de toda la vida es demasiado fácil enseñar a los niños conductas adecuadas o "lindas" para su edad, pero que serán percibidas como inapropiadas en el futuro. Esto implicaria que el niño tenga que aprender nuevas conductas unos años después. Por ejemplo, muchos signos para ir al baño que son apropiados para los niños pequeños pueden ser ofensivos $o$ aun indecentes cuando son usados por un adulto.

La prioridad para el preescolar es con frecuencia aprender las principales habilidades básicas y una orientación hacia el trabajo. Conforme el niño crece se vuelve cada vez más importante dirigir el entrenamiento hacia las habilidades funcionales que le serán necesarias en su vida futura. Estas habilidades con frecuencia son indispensables para vivir en la forma más independiente y significativa posible de adulto.

Con el propósito de tener una vida independiente, a cada niño se le debe facilitar el aprendizaje de por lo menos las siguientes habilidades (no es posible presentar una lista completa de las habilidades básicas. Lo que se incluye en la lista depende de los intereses, fortalezas y necesidades del individuo; asi como de las prioridades de la familia):

1. Habilidades de comunicación: A cada niño se le debe dar la posibilidad de expresar sus necesidades y pensamientos, asi como la de comprender a los demás. Obviamente, la comunicación tiene que ser adaptada a sus habilidades. Tomando en cuenta la discapacidad, el lenguaje hablado rara vez es la primera opción, ni para la comunicación expresiva, ni para la receptiva. $\mathrm{Sin}$ embargo, las ayudas visuales en la comunicación con frecuencia promueven la habilidad de usar el lenguaje hablado.

2. Habilidades de autoayuda: Para el niño o el adulto con retraso mental severo, tener suficientes habilidades de autoayuda puede definirse como ser capaz de ir al baño, asearse, alimentarse, etc., en la forma más independiente posible. Para los adultos más capaces las habilidades de autoayuda incluyen una variedad de tareas domésticas, por ejemplo cocinar y hacer limpieza.
3. Habilidades vocacionales: En los primeros años escolares no parece ser muy significativo pensar qué tipo de empleo realizará el niño en el futuro. Pero es realmente significativo darle al niño una amplia educación en habilidades relacionadas con el trabajo que puedan aplicarse a oportunidades laborales potenciales. Además, el niño tiene que aprender conductas de trabajo apropiadas, tales como mantener un ritmo constante en el trabajo, terminar las tareas y no molestar a otros. Esto es esencial para cualquier empleo o actividad diaria en el futuro.

4. Habilidades recreativas: Lograr que el tiempo libre sea significativo resulta fundamental cuando se habla de calidad de vida. Es demasiado desgastante para muchos padres de hijos con autismo no poder dejar a sus hijos sin estrecha supervisión. Desde el principio, tanto los padres como la familia se beneficiarán del aprendizaje del niño para involucrarse en el juego $u$ otras actividades recreativas por si mismo. En una perspectiva de toda la vida es importante apoyar al niño a que desarrolle pasatiempos en los que las personas puedan participar a lo largo de la vida.

5. Habilidades comunitarias: Para que el niño sea capaz de participar en la vida de la comunidad necesita aprender cómo interactuar y comportarse en público de una manera adecuada, pero no necesariamente de manera normal. Las habilidades más importantes a aprender son definidas en forma individual.

No he incluido las habilidades sociales en la lista. El deterioro cualitativo en la interacción social es parte del criterio diagnóstico e influye cada contacto con los demás. A lo largo de la vida una persona con autismo se encontrará con otras personas en casa, en la escuela, en el trabajo, durante su tiempo de ocio, en el camión, en el supermercado, etc. Las personas con autismo en verdad necesitan habilidades sociales, pero desde mi punto de vista éstas tienen que ser aprendidas en situaciones especificas. Nosotros, que trabajamos en el área del autismo, sabemos que las personas con el trastorno tienen grandes dificultades generalizando tareas de ejecución a través de los contextos. Dos caracteristicas muy comunes de la atención en el autismo hacen todavia más complicado que ellos puedan generalizar situaciones sociales. Comúnmente, las personas con autismo se enfocan en los detalles y tienen problemas para distinguir entre los estimulos relevantes y 
los irrelevantes. Esto implica que cada situación social puede parecer nueva para muchas personas con autismo. De aqui que aprender habilidades sociales fuera de contexto resulte extremadamente dificil. Probablemente no sea tan dificil aprender en una situación de entrenamiento, pero utilizar ese conocimiento en una situación de la vida real en la comunidad es muy complicado para las personas con autismo. Desde un punto de vista funcional es más útil aprender habilidades sociales en situaciones especificas.

\section{Conclusión}

Al trabajar con niños con autismo, es esencial tener una perspectiva de toda la vida. Esto sólo es posible si el trabajo esta fundamentado en un verdadero conocimiento del autismo, un profundo interés y respeto para cada niño, así como una sincera colaboración con las familias y entre los profesionales. Sólo asi podremos ofrecer al niño los medios para que pueda convertirse en un adulto viviendo una vida con la mejor calidad posible.

\section{Referencias}

Schopler, E., Mesibov, G.B., Shigley, R.H. \& Bashford, A. (1984). Helping Autistic Children Through Ther Parents. En Schopler, E. \& Mesibov, G.B. (eds.). The Effects of Autism on the Family. Plenum Press, Nueva York.

Simmons III, J.Q. \& Lovaas O.I. (1969). Use of Pain and Punishment as Treatment Techniques with Childhood Schizophrenics. American Journal of Psychotherapy

Sinclair, J. (1992). Bridging the Gaps: An Inside- Out View of Autism (or you know what I don't know?) en Scho- pler, E. \& Mesibov, G.B. (eds). High Performance Individuals with Autism. Plenum Press, Nueva York.

Sinclair, J. (1993). Don't Mourn for Us www.autistics.org/ library/dontmourn.html Primera publicación en Our Voice, newsletter of Autism Network International, Volumen 1, número $3,1993$.

Swedish Code of Statutes SFS 1993:387 Acto referente al Apoyo y Servicio para Personas con cierto grado de deterioro funcional. 